\title{
SIMULACIÓN CON PACIENTES ESTANDARIZADOS EN CIENCIAS DE LA SALUD: UNA REVISIÓN SISTEMÁTICA
}

\author{
HUMAN PATIENT SIMULATION IN HEALTH SCIENCES: A SYSTEMATIC REVIEW
}

\section{Jose Antonio Merchán-Baeza', Manuel González-Sánchez² y David Pérez-Cruzado³}

\begin{abstract}
RESUMEN
Introducción: Los métodos de enseñanza utilizados en terapia ocupacional son numerosos y podrían clasificarse en dos grupos: métodos de enseñanza tradicionales y alternativos. La simulación es un método alternativo que permite el aprendizaje autónomo y el desarrollo de habilidades de pensamiento crítico de los estudiantes durante su participación activa en una práctica segura. Objetivo: Analizar la efectividad del aprendizaje a través de la simulación con pacientes estandarizados frente a la enseñanza tradicional en ciencias de la salud. Método: La estrategia de búsqueda fue: ("Patient Simulation" OR "Role Playing" OR "Simulation Scenario" OR "Simulation Education") AND ("Program Evaluation" OR Effectiveness) AND (Student OR Postgraduate). El análisis de validez interna de los artículos incluidos en la revisión se realizó mediante la escala PEDro. Resultados: Se incluyeron catorce estudios y las variables evaluadas se organizaron en cuatro categorías: adquisición de conocimiento, aprendizaje de la práctica clínica, autoeficacia y desarrollo de habilidades de comunicación. Las muestras estuvieron compuestas por estudiantes de las profesiones de la salud y las simulaciones tuvieron una duración de entre 1o y 4o minutos por sesión (4-24 sesiones). Conclusión: No se encontraron diferencias significativas entre ambas metodologías en la adquisición de habilidades prácticas por parte de los estudiantes. Aun así, la visualización de videos explicativos al principio y el resumen al final del proceso de simulación, han demostrado ser etapas fundamentales para mejorar la efectividad de este método de enseñanza. Futuros estudios son necesarios para analizar los posibles beneficios de la simulación en la titulación de terapia ocupacional.
\end{abstract}

\section{PALABRAS CLAVE}

Ciencias de la Salud, Comunicación de la Salud, Simulación de Paciente, Práctica profesional, Evaluación educacional.

\begin{abstract}
Introduction: Teaching methods used in occupational therapy are numerous and could be classified into two groups: traditional and alternative teaching methods. Simulation is an alternative method that allows autonomous learning while developing the critical thinking skills of students during their active participation in safe practice. Objective: To analyze the effectiveness of learning through simulation with standardized patients compared to traditional teaching in health sciences. Method: The search strategy was: ("Patient Simulation" OR "Role Playing" OR "Simulation Scenario" OR "Simulation Education") AND ("Program Evaluation" OR Effectiveness) AND (Student OR Postgraduate). The analysis of internal validity of the articles included in the review was performed using the PEDro scale. Results: Fourteen studies were included and variables measured were organized into four categories: acquisition of knowledge, learning of clinical practice, self-efficacy and development
\end{abstract}

1 Doctor en Ciencias de la Salud, Universidad de MálagaTerapeuta Ocupacional. Grupo de investigación Methodology, Methods, Models and Outcomes of Health and Social Sciences $\left(\mathrm{M}_{3} \mathrm{O}\right)$, Facultad de Ciencias de la Salud y Bienestar, Universidad de Vic-Universidad Central de Cataluña (UVIC-UCC), o850o Vic, España. ORCID ID: oooo-0oo2-6893-952X. Carrer de la Sagrada Familia 7, o850o, Vic (Barcelona), España. josan.merchan@uvic.cat / +34 9388861222

2 Doctor en Ciencias de la Salud, Universidad de Málaga. Fisioterapeuta. Universidad de Málaga, Departamento de fisioterapia, Instituto de Investigación Biomédica de Málaga (IBIMA), 29071, Málaga, España. ORCID ID: oooo-ooo3-3993-5092 mgsa23@uma.es /+34 951952854

3 Doctor en Ciencias de la Salud, Universidad de Málaga.Terapeuta Ocupacional. Departamento de Terapia Ocupacional, Universidad Católica San Antonio de Murcia, Murcia. ORCID ID: oooo-0o01-7952-0831 dpcruzado@ucam.edu 
of communication skills. The samples were health professions students and simulations had a duration from 10 to 40 minutes per session (4-24 sessions). Conclusion: No significant differences were found between both methodologies in the acquisition of practical skills by students. Even so, the visualization of explanatory videos at the beginning and the summary at the end of the simulation process have proven to be fundamental steps to improve the effectiveness of this teaching method. Future studies are needed to analyze the possible benefits of simulation in the occupational therapy degree.

\section{KEYWORDS}

Health Sciences, Health communication, Patient simulation, Professional practice, Educational Measurement.

\section{INTRODUCCIÓN}

Los métodos de enseñanza utilizados hoy en día en ciencias de la salud son numerosos y variados, y se pueden clasificar en dos grupos principales. Los métodos de enseñanza tradicionales (Andrighetti et al., 2012; Elizondo-Omaña et al., 2004; McParland et al., 2004) y alternativos (Bingham et al., 2015; Guarino et al., 2014; Jansson et al., 2014).

La simulación es un método alternativo de enseñanza que permite el aprendizaje autónomo de los estudiantes mediante el desarrollo de habilidades de pensamiento crítico durante su participación activa en una práctica segura, sin la posibilidad de causar daño a pacientes reales(Andrighetti et al., 2012; Stefaniak \& Turkelson, 2014). Por lo general, comienza con una breve introducción informativa que se presenta al alumno en un escenario en el que debe realizar una intervención simulada con un maniquí o un actor que interpreta una patología y una situación dada (Andrighetti et al., 2012; Aper et al., 2012). Finalmente se lleva a cabo la sesión informativa, en la que el alumno realiza una autoevaluación, recibe la retroalimentación del experto y/o del paciente simulado o el compañero observador (Elley et al., 2012; Gordon et al., 2013; Jansson et al., 2014). A veces, un debate con los estudiantes les permite enfocarse en sus experiencias, tanto positivas como negativas (Koponen et al., 2012). Este método también permite a los estudiantes experimentar o manejar situaciones complejas de la práctica clínica (Stefaniak \& Turkelson, 2014).
La simulación de maniquí es muy utilizada en áreas específicas de medicina y enfermería que requieren que el paciente simulado muestre ciertos síntomas que los pacientes reales no pueden simular (Bingham et al., 2015; Hong et al., 2014). La fidelidad en estas simulaciones es muy importante y se refiere al nivel en el que la situación se asemeja a la realidad (Andrighetti et al., 2012). Sin embargo, en estas disciplinas existen conceptos y habilidades de comunicación directa con el paciente que deben llevarse a cabo con actores o compañeros de clase que simulen una situación determinada, ya que estas no pueden ser realizadas con maniquíes sobre todo en algunas disciplinas como la terapia ocupacional, en la cual es necesaria la participación activa del usuario (Becker et al., 2006; Kesten, 2011; Schlegel et al., 2012; Zavertnik et al., 2010).

Aunque hay algunos estudios que comparan este método de enseñanza con otros, la evidencia existente no ha sido evaluada y clasificada de una manera que permita identificar las limitaciones de este método de enseñanza. El objetivo de esta revisión sistemática es, por lo tanto, determinar la efectividad de la simulación con paciente estandarizado sobre los métodos de enseñanza tradicionales en diferentes disciplinas de las ciencias de la salud. 


\section{MÉTODO}

\section{Diseño}

Se realizó una revisión sistemática sobre la aplicación de la simulación con pacientes estandarizados frente a la metodología tradicional siguiendo los estándares de PRISMA (Moher et al., 2009). El protocolo de esta revisión se registró en el International Prospective Register of Systematic Reviews (PROSPERO): CRD42O15O19852.

\section{Estrategia de búsqueda}

La búsqueda bibliográfica se realizó de mayo a junio de 2019 utilizando las bases de datos Pubmed, Scopus y Scielo. Los primeros términos de búsqueda se obtuvieron de $\mathrm{MeSH}$ y se agregaron otros términos para refinar la búsqueda y proporcionar la cadena de búsqueda final: ("Patient Simulation" OR "Role Playing" OR "Simulation Scenario" OR "Simulation Education") AND ("Program Evaluation" OR Effectiveness) AND (Student OR Postgraduate). Además, los estudios obtenidos se filtraron para ser ensayos clínicos aleatorizados (ECA), ensayos clínicos (EC), informes de casos (IC), ensayos clínicos controlados (ECC) y cuasiexperimentales.

Dos investigadores cegados (DPC y JAMB) seleccionaron de forma independiente en dos etapas los estudios encontrados en la búsqueda realizada. En la primera etapa se evaluaron los títulos y resúmenes de los estudios, y en la segunda etapa se evaluó el texto completo de los artículos restantes. Un evaluador independiente (MGS) fue la persona responsable de los estudios en los que hubo cierta discrepancia de criterios entre los dos investigadores.

\section{Criterios de inclusión y exclusión}

Se incluyeron ECA, EC, IC, ECC y estudios cuasiexperimentales en los que se había utilizado la simulación con paciente estandarizado. Se incluyeron estudios en inglés, español, francés, portugués e italiano (todos en idioma original) y donde la muestra eran estudiantes de ciencias de la salud, incluidos estudiantes de posgrado. Se excluyeron los estudios con un punto de corte inferior a cinco puntos en la escala de validez interna PEDro (Moseley et al., 2002).

\section{Análisis de validez interna}

El análisis de la validez interna de los artículos incluidos en la revisión se realizó utilizando la escala PEDro, cuyo ICC es de o.68 (IC 95\% = 0.57-0.76) (Maher et al., 2003). Esta escala consta de 11 ítems y valoraron los criterios de selección, designación de muestra aleatoria, asignación oculta, similitud de los grupos, ceguera de los participantes, terapeutas y evaluadores, recopilación de resultados clave del 85 por ciento de los participantes, presentación de datos para todos los participantes, comparación entre grupos y medidas de variabilidad, aunque en la puntuación total se excluye el primer ítem, por lo que esta puede encontrarse en o y 10 puntos. Dos investigadores (DPC у ЈАMB) evaluaron de forma independiente los estudios incluidos con la escala PEDro.

\section{Características extraídas de los estudios}

El diseño del estudio, el perfil y el número de participantes, los grupos de estudio, los detalles de la simulación, las variables de resultado, el momento de las medidas y los instrumentos de medición se extrajeron de los artículos incluidos en la revisión y se mostraron en la tabla 2. Además, en la tabla 3 se extrajeron los valores para todas las variables de resultado analizadas en cada uno de los estudios.

\section{Resultados}

A partir de la estrategia de búsqueda inicial y con los filtros marcados se obtuvieron 78 artículos. Veinte de estos fueron excluidos después de leer el título debido a su falta de conexión con el tema. De los 46 artículos restantes, 22 fueron excluidos después de revisar el resumen y otros 16 después de revisar el artículo completo. Se excluyeron estos 38 estudios ya que la población objetivo no eran estudiantes de ciencias de la salud, porque se usaron maniquíes en la simulación o porque no tenían ninguno de los criterios de inclusión definidos al comienzo de esta revisión sistemática. Los 16 estudios restantes se incluyeron en el análisis de validez interna realizado mediante la escala PEDro. Después de realizar 
FIGURA 1 DIAGRAMA DE FLUJO DE LA SELECCIÓN DE ESTUDIOS

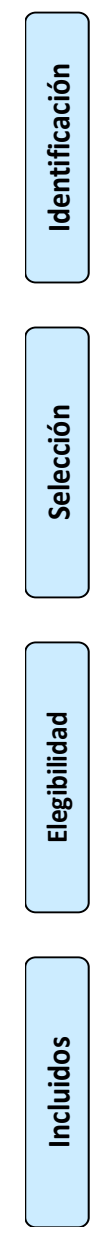

Artículos identificados tras la búsqueda en bases de datos $(\mathrm{n}=78)$
Artículos identificados a través de otras fuentes $(\mathrm{n}=0)$

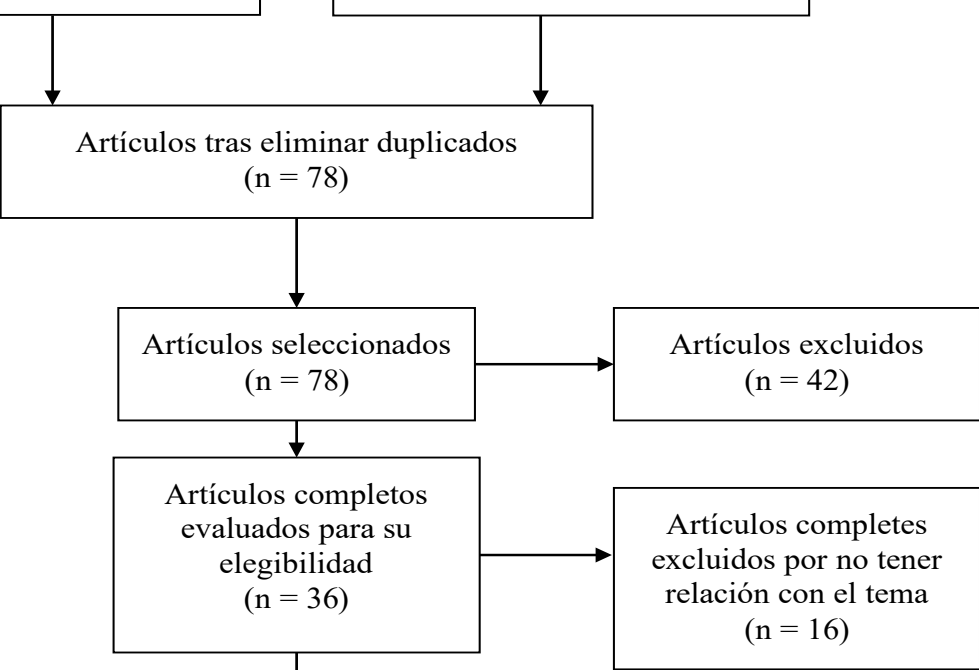

Artículos incluidos en la síntesis cualitativa $(\mathrm{n}=20)$

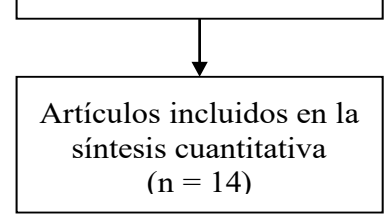

este análisis y establecer un límite igual o superior a cinco puntos, se incluyeron 14 estudios en esta revisión sistemática. Los detalles de la selección se muestran en la figura 1.

La Tabla 1 muestra la puntuación obtenida por el artículo seleccionado para el análisis de validez interna realizado mediante la escala PEDro. Para este análisis se incluyeron 20 artículos que alcanzaron los prefiltros. Después de analizar la puntuación final de cada uno de los estudios, los autores establecieron un límite igual o superior a 5 puntos para decidir qué artículos se incluirían en la revisión sistemática. Ante este límite, se incluyeron 14 de los 20 estudios analizados.
La Tabla 2 muestra los valores de las variables extraídas en todos los estudios. Doce de los estudios analizados fueron ECA y dos fueron cuasiexperimentales. Las muestras en todos los estudios consistieron en estudiantes de enfermería o medicina, y simulaciones realizadas con pacientes con una duración de entre 10 y 40 minutos por sesión, sin un número establecido de sesiones (van de 4 a 24 sesiones). Las variables medidas se organizaron en cuatro categorías: adquisición de conocimiento, aprendizaje de la práctica clínica, autoeficacia y desarrollo de habilidades de comunicación. 
TABLA 1 ANÁLISIS DE VALIDEZ INTERNA (ESCALA PEDRO)

\begin{tabular}{|c|c|c|c|c|c|c|c|c|c|c|c|}
\hline Autores & 2 & 3 & 4 & 5 & 6 & 7 & 8 & 9 & 10 & 11 & Total \\
\hline Aper L. et al. & - & - & - & - & - & - & $\bullet$ & - & - & - & 6 \\
\hline Becker KL. et al. & $\bullet$ & - & $\bullet$ & - & - & - & - & $\bullet$ & $\bullet$ & $\bullet$ & 5 \\
\hline Brady S. et al. & $\bullet$ & $\bullet$ & - & - & - & - & $\bullet$ & $\bullet$ & $\bullet$ & $\bullet$ & 6 \\
\hline Chin et al. & $\bullet$ & - & $\bullet$ & - & - & - & $\bullet$ & $\bullet$ & $\bullet$ & $\bullet$ & 6 \\
\hline Elley CR. Et al. & • & $\bullet$ & $\bullet$ & - & $\bullet$ & - & $\bullet$ & - & $\bullet$ & - & 6 \\
\hline Janjua A. et al. & - & $\bullet$ & $\bullet$ & - & $\bullet$ & - & - & - & $\bullet$ & $\bullet$ & 6 \\
\hline Jansson $\mathrm{M}$ et al. & $\bullet$ & $\bullet$ & $\bullet$ & - & - & $\bullet$ & - & - & $\bullet$ & - & 5 \\
\hline Kessler CS. et al. & $\bullet$ & $\bullet$ & $\bullet$ & - & - & - & $\bullet$ & $\bullet$ & $\bullet$ & $\bullet$ & 8 \\
\hline Kesten KS. & $\bullet$ & $\bullet$ & $\bullet$ & - & - & - & $\bullet$ & $\bullet$ & $\bullet$ & $\bullet$ & 7 \\
\hline Kim S. et al. & $\bullet$ & $\bullet$ & $\bullet$ & - & - & - & $\bullet$ & $\bullet$ & $\bullet$ & $\bullet$ & 7 \\
\hline Knowles C. et al. & $\bullet$ & $\bullet$ & $\bullet$ & - & - & - & $\bullet$ & $\bullet$ & - & - & 5 \\
\hline Koponen J. et al. & $\bullet$ & - & $\bullet$ & - & - & - & $\bullet$ & $\bullet$ & $\bullet$ & $\bullet$ & 6 \\
\hline Schlegel C. et al. & $\bullet$ & $\bullet$ & $\bullet$ & - & - & - & - & $\bullet$ & $\bullet$ & - & 5 \\
\hline Zavertnik JE. et al. & - & - & $\bullet$ & - & - & - & $\bullet$ & $\bullet$ & $\bullet$ & - & 5 \\
\hline
\end{tabular}

- $\mathrm{Si}-\mathrm{No}$ 


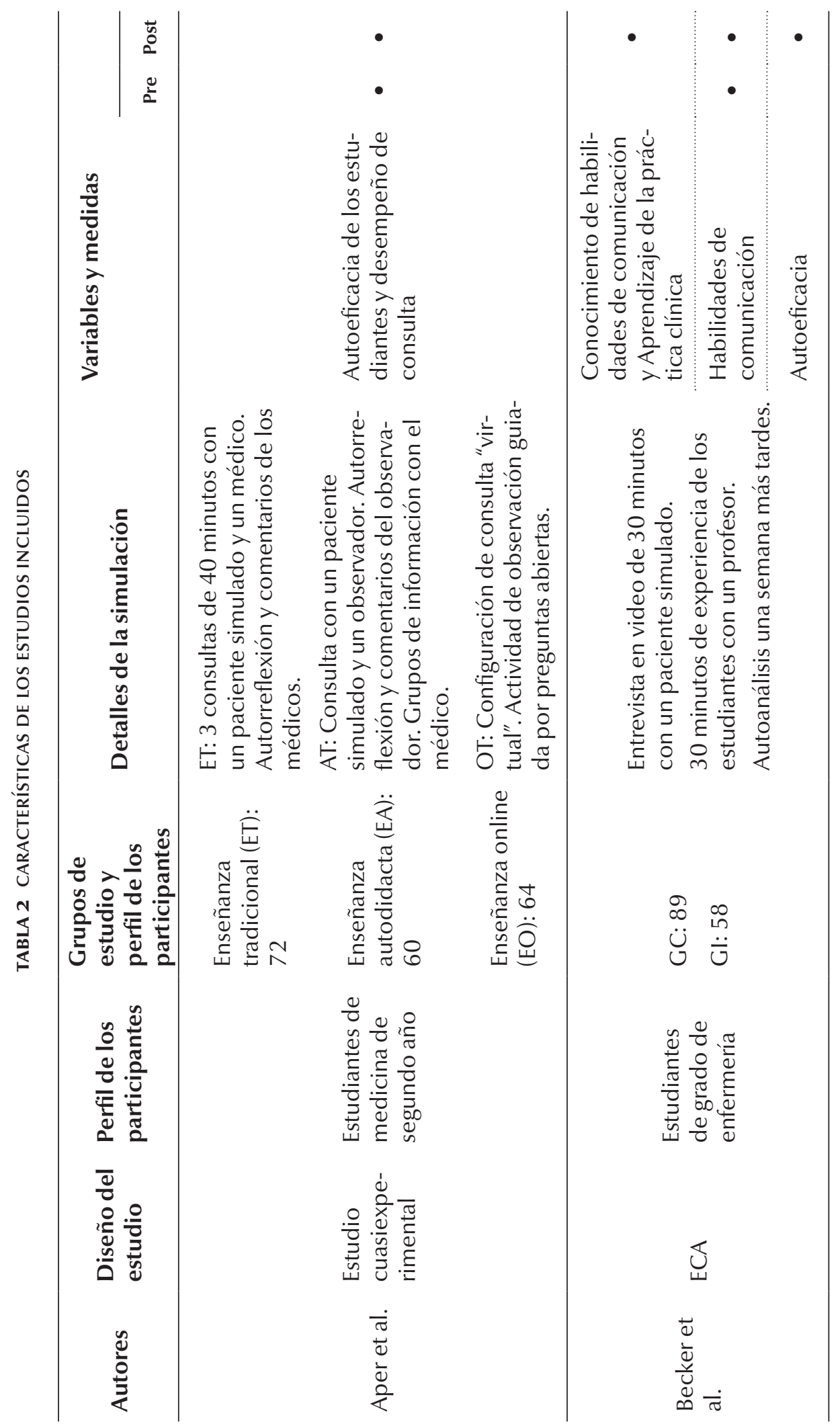




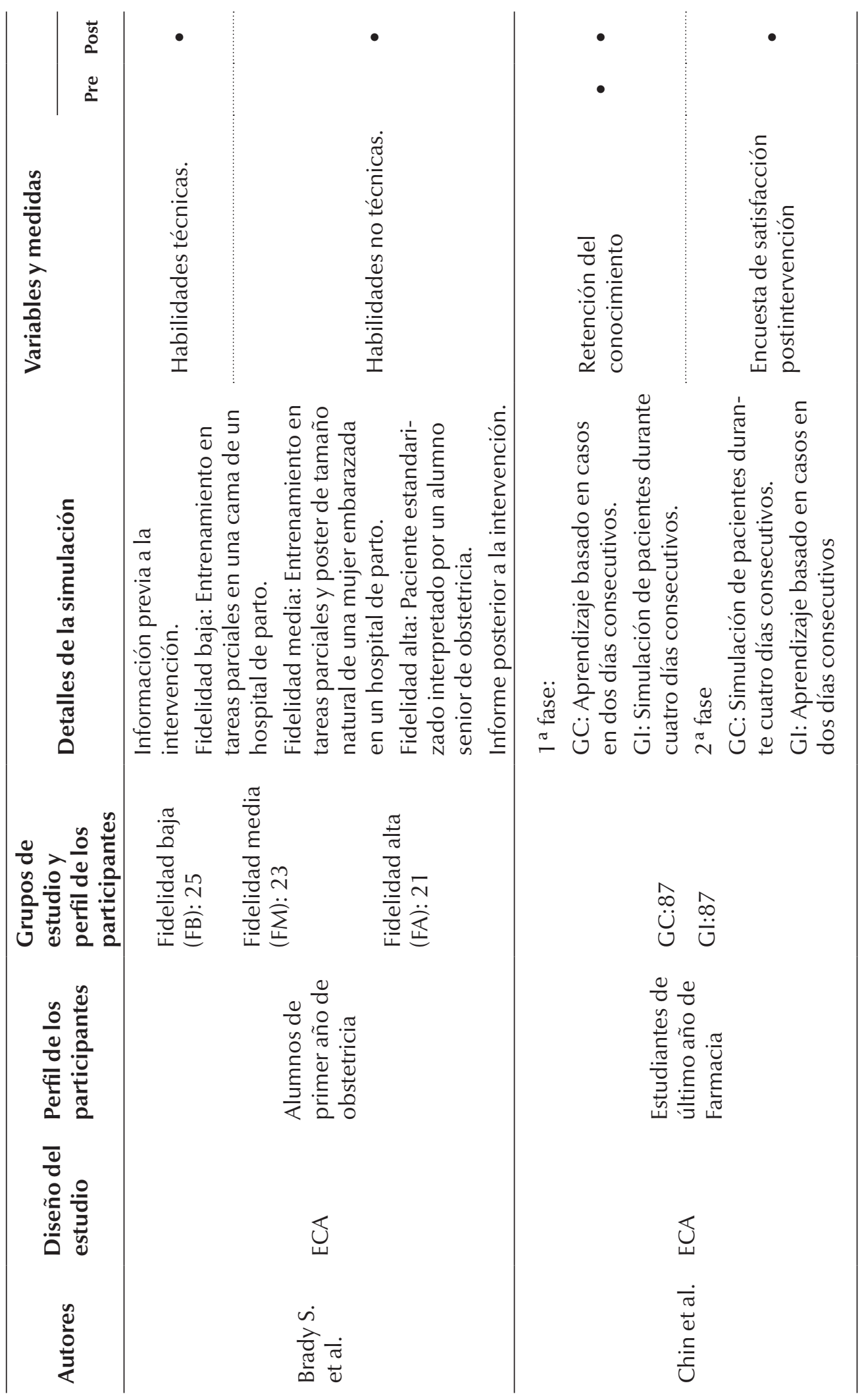




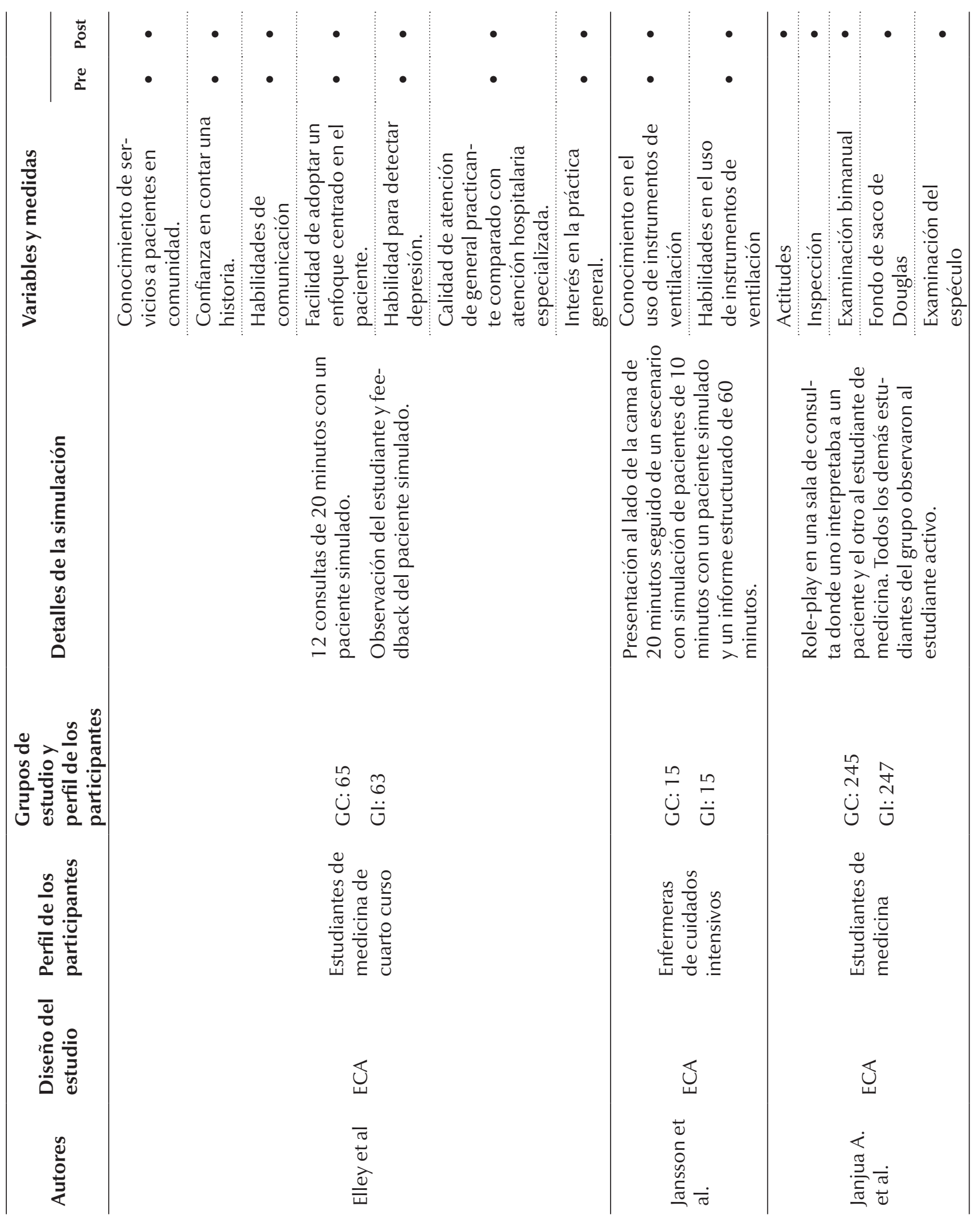




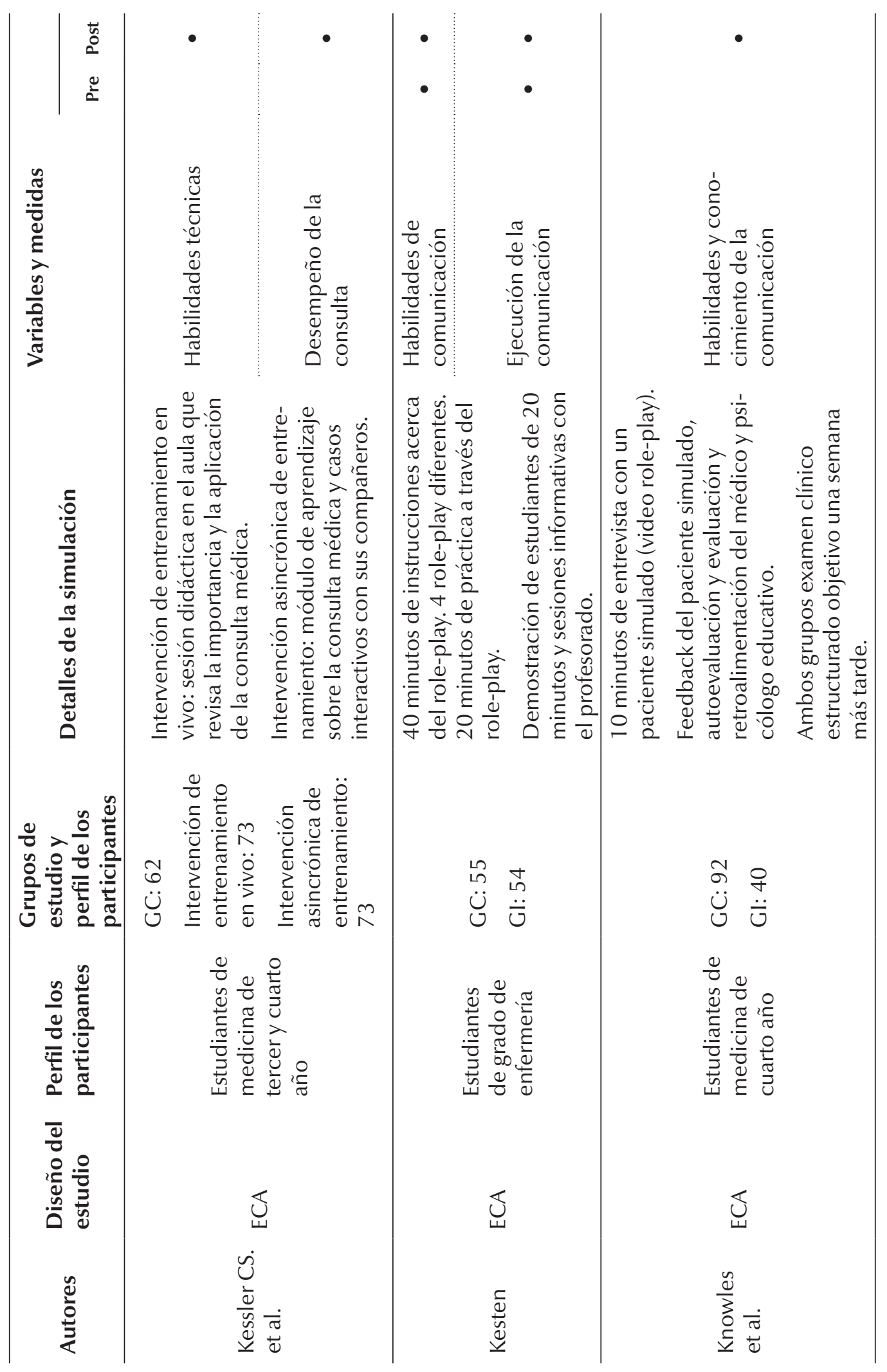




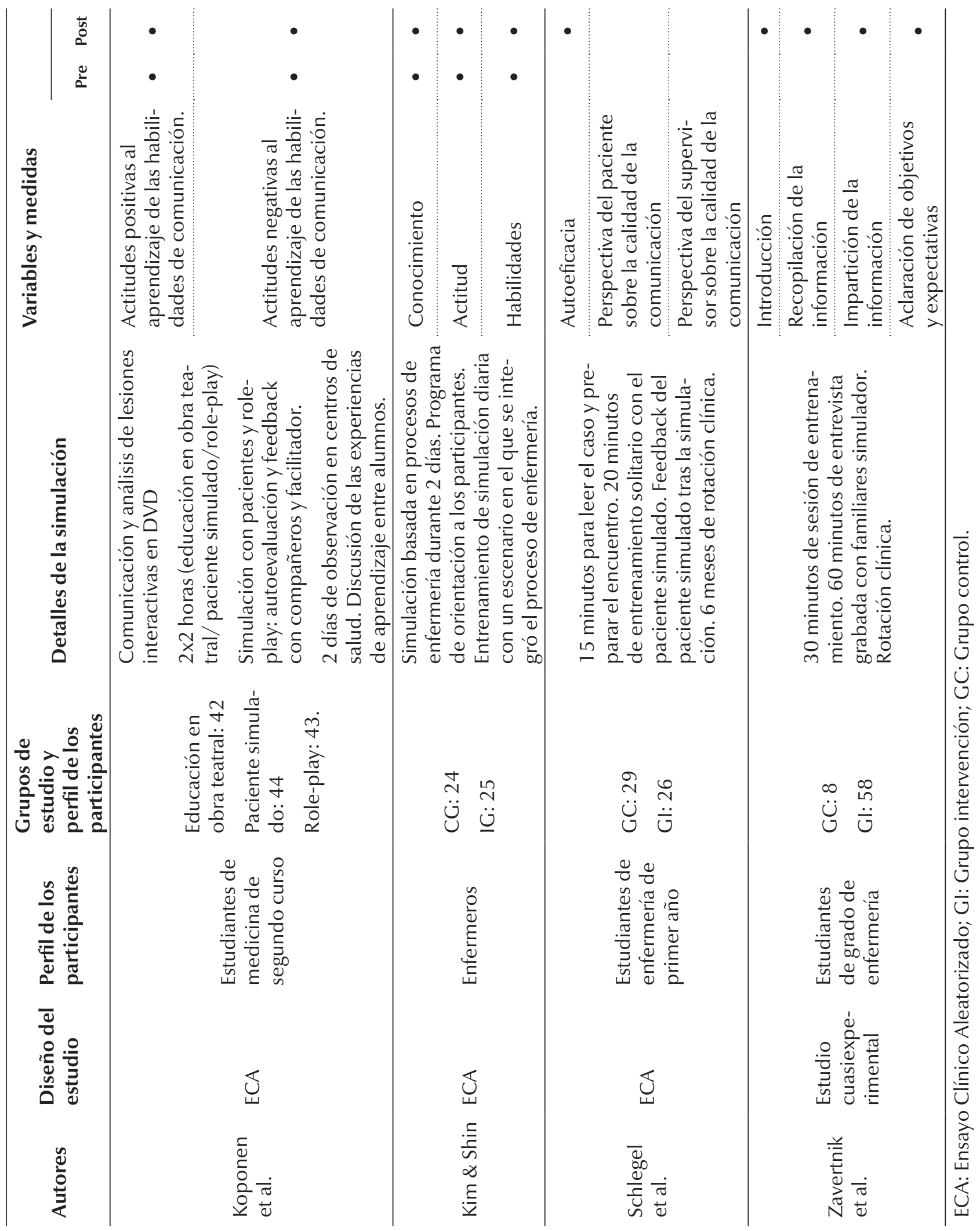


La Tabla 3 muestra los resultados de los estudios incluidos en esta revisión sistemática. Los efectos intragrupo se midieron en cinco estudios (Aper et al., 2012; Becker et al., 2006; Hong et al., 2014; Janjua et al., 2017; Koponen et al., 2012) y se descubrió que el grupo que realizó la simulación de pacientes estandarizados mostró mejoras en la adquisición de conocimiento (Becker et al., 2006; Kim \& Shin, 2016), self-efficacy (Aper et al., 2012; Janjua et al., 2017), desarrollo de habilidades comunicativas (Koponen et al., 2012) y aprendizaje de la práctica clínica (Janjua et al., 2017; Kim \& Shin, 2016). Las diferencias intergrupo se midieron en todos los estudios, excepto en el de Aper et al. Se encontraron diferencias entre los grupos de intervención y control en la adquisición de conocimiento (Brady et al., 2015; Jansson et al., 2014; Kesten, 2011; Kim \& Shin, 2016; Lee Chin et al., 2014), aprendizaje de la práctica clínica (Brady et al., 2015; Elley et al., 2012; Janjua et al., 2017; Jansson et al., 2014; Kim \& Shin, 2016), desarrollo de habilidades comunicativas (Elley et al., 2012; Kessler et al., 2015; Kesten, 2011; Koponen et al., 2012; Schlegel et al., 2012; Zavertnik et al., 2010), y autoeficacia (Aper et al., 2012; Janjua et al., 2017).

TABLA 3 RESULTADOS EXTRAIDOS DE LOS ESTUDIOS INCLUIDOS

\begin{tabular}{|c|c|c|c|c|c|c|c|c|}
\hline \multirow[b]{2}{*}{ Autor(es) } & \multirow[b]{2}{*}{ Variables } & & \multirow{2}{*}{$\begin{array}{l}\text { Preinter- } \\
\text { vención } \\
\pm \mathrm{SD}\end{array}$} & \multirow{2}{*}{$\begin{array}{l}\text { Postinter- } \\
\text { vención } \\
\pm \mathrm{SD}\end{array}$} & \multicolumn{2}{|c|}{$\begin{array}{c}\text { Diferencias } \\
\text { significativas }\end{array}$} & \multicolumn{2}{|c|}{ Cohen's d (post) } \\
\hline & & & & & $\begin{array}{l}\text { Intra } \\
\text { grupo }\end{array}$ & $\begin{array}{l}\text { Inter } \\
\text { grupo }\end{array}$ & $\begin{array}{l}\text { Inter } \\
\text { grupo }\end{array}$ & $\begin{array}{l}\text { Intra } \\
\text { grupo } \\
\text { (GI) }\end{array}$ \\
\hline \multirow{9}{*}{ Aper et al. } & \multirow{6}{*}{$\begin{array}{l}\text { Autoeficacia de } \\
\text { los estudiantes }\end{array}$} & \multirow{3}{*}{8 sub-ítems } & ET: $6.56 \pm 0.89$ & ET: $6.54 \pm 0.99$ & \multirow{6}{*}{ - } & $\mathrm{ET}: \circ$ & $\begin{array}{c}\text { EA vs ET: } \\
0.45\end{array}$ & \multirow{3}{*}{0.42} \\
\hline & & & EA: $6.58 \pm 0.97$ & EA: $6.98 \pm 0.94$ & & EA: • & \multirow{2}{*}{$\begin{array}{c}\text { EA vs EO: } \\
0.44\end{array}$} & \\
\hline & & & EO: $6.36 \pm 0.76$ & EO: $6.60 \pm 0.79$ & & EO: $\circ$ & & \\
\hline & & \multirow{3}{*}{9 ítems } & ET: $6.17 \pm 1.02$ & ET: $6.29 \pm 1.05$ & & ET: ○ & \multirow[t]{2}{*}{$\begin{array}{c}\text { EA vs ET: } \\
0.39\end{array}$} & \multirow{3}{*}{0.58} \\
\hline & & & EA: $5.98 \pm 1.36$ & EA: $6.75 \pm 1.27$ & & EA: • & & \\
\hline & & & EO: $5.88 \pm 1.12$ & EO: $5.84 \pm 1.13$ & & EO: $\circ$ & 0.76 & \\
\hline & \multirow{3}{*}{\multicolumn{2}{|c|}{ Desempeño de la consulta }} & ET: 9.96 \pm 2.14 & ET: $11.56 \pm 2.41$ & \multirow{3}{*}{-} & $\mathrm{ET}: \bullet$ & \multirow{3}{*}{$\begin{array}{l}\text { EA vs ET: } \\
-0.43 \\
\text { EA vs EO: } \\
-0.52 \\
\end{array}$} & \multirow{3}{*}{0.08} \\
\hline & & & EA: $10.29 \pm 1.99$ & EA: $10.48 \pm 2.57$ & & EA: $\circ$ & & \\
\hline & & & EO: $10.42 \pm 2.84$ & EO: $11.85 \pm 2.62$ & & EO: • & & \\
\hline \multirow{5}{*}{$\begin{array}{l}\text { Becker } \\
\text { et al. }\end{array}$} & \multirow{2}{*}{\multicolumn{2}{|c|}{ Habilidades de comunicación }} & GC: $15.54 \pm 2.26$ & GC: $16.36 \pm 2.02$ & \multirow{2}{*}{ ० } & GI: • & \multirow{2}{*}{0.03} & \multirow{2}{*}{0.70} \\
\hline & & & GI: $14.79 \pm 2.61$ & GI: $16.42 \pm 1.86$ & & GC: • & & \\
\hline & \multirow{2}{*}{$\begin{array}{l}\text { Conocimiento de } \\
\text { habilidades de } \\
\text { comunicación y } \\
\text { Aprendizaje de la } \\
\text { práctica clínica }\end{array}$} & $\begin{array}{l}\text { Formulario de califi- } \\
\text { caciones de relacio- } \\
\text { nes interpersonales } \\
\text { del paciente }\end{array}$ & - & $\begin{array}{l}\text { GC: } 14.10 \pm 4.72 \\
\text { GI: } 14.26 \pm 6.23\end{array}$ & 0 & - & 0.03 & - \\
\hline & & $\begin{array}{l}\text { Checklist estandari- } \\
\text { zado post encuentro } \\
\text { con el paciente }\end{array}$ & - & $\begin{array}{l}\text { GC: } 0.52 \pm 0.20 \\
\text { Gl: } 0.61 \pm 0.17\end{array}$ & $\circ$ & - & 0.48 & - \\
\hline & \multicolumn{2}{|l|}{ Autoeficacia } & - & GI: $18.42 \pm 3.88$ & - & - & - & - \\
\hline
\end{tabular}




\begin{tabular}{|c|c|c|c|c|c|c|c|}
\hline \multirow[b]{2}{*}{ Autor(es) } & \multirow[b]{2}{*}{ Variables } & \multirow{2}{*}{$\begin{array}{l}\text { Preinter- } \\
\text { vención } \\
\quad \pm \mathrm{SD}\end{array}$} & \multirow{2}{*}{$\begin{array}{l}\text { Postinter- } \\
\text { vención } \\
\pm S D\end{array}$} & \multicolumn{2}{|c|}{$\begin{array}{l}\text { Diferencias } \\
\text { significativas }\end{array}$} & \multicolumn{2}{|c|}{ Cohen's d (post) } \\
\hline & & & & $\begin{array}{l}\text { Intra } \\
\text { grupo }\end{array}$ & $\begin{array}{l}\text { Inter } \\
\text { grupo }\end{array}$ & $\begin{array}{l}\text { Inter } \\
\text { grupo }\end{array}$ & $\begin{array}{c}\text { Intra } \\
\text { grupo } \\
\text { (GI) }\end{array}$ \\
\hline \multirow{8}{*}{$\begin{array}{l}\text { Brady S. } \\
\text { et al. }\end{array}$} & \multirow{4}{*}{ Habilidades técnicas } & \multirow{4}{*}{-} & FB: $19.9 \pm 6.30$ & \multirow{4}{*}{$\begin{array}{l}\text { FA vs } \\
\text { FB: } \\
\text { FA vs } \\
\text { FM: • }\end{array}$} & \multirow{4}{*}{-} & \multirow{4}{*}{$\begin{array}{c}\text { FA vs FB: } \\
0.90 \\
\text { FA vs FM: } \\
0.71\end{array}$} & \multirow[t]{3}{*}{-} \\
\hline & & & FM: & & & & \\
\hline & & & $20.9 \pm 7.10$ & & & & \\
\hline & & & FA: $26.0 \pm 7.1$ & & & & - \\
\hline & \multirow{4}{*}{ Habilidades no técnicas } & \multirow{4}{*}{-} & FB: $29.9 \pm 9.8$ & \multirow{4}{*}{$\begin{array}{l}\text { FA vs } \\
\text { FB: • } \\
\text { FA vs } \\
\text { FM: • }\end{array}$} & \multirow{4}{*}{-} & \multirow{4}{*}{$\begin{array}{c}\text { FA vs FB: } \\
0.80 \\
\text { FA vs FM: } \\
0.79\end{array}$} & \multirow{4}{*}{$\begin{array}{l}- \\
-\end{array}$} \\
\hline & & & FM: & & & & \\
\hline & & & $29.7 \pm 10.6$ & & & & \\
\hline & & & FA: $38.1 \pm 10.5$ & & & & \\
\hline \multirow{12}{*}{ Chin et al. } & \multirow{6}{*}{ Retención del conocimiento } & & FASE 1 & \multirow{6}{*}{$\begin{array}{l}\text { Fase } \\
1: ॰ \\
\text { Fase } \\
2: \bullet\end{array}$} & \multirow{6}{*}{-} & \multirow{6}{*}{$\begin{array}{c}\text { Fase 1: } \\
-0.17 \\
\text { Fase 2: } \\
0.32\end{array}$} & \multirow{6}{*}{$\begin{array}{c}\text { Fase } \\
1: \\
0.88 \\
\text { Fase } \\
2: \\
1.95\end{array}$} \\
\hline & & GC: $87.70 \pm 8.4$ & $\begin{array}{c}\text { GC: } \\
93.50 \pm 6.90\end{array}$ & & & & \\
\hline & & $\begin{array}{c}\text { Gl: } \\
84.10 \pm 11.00\end{array}$ & $\begin{array}{c}\text { GI: } \\
92.30 \pm 7.20\end{array}$ & & & & \\
\hline & & FASE 2 & FASE 2 & & & & \\
\hline & & $\begin{array}{c}\text { GC: } \\
57.60 \pm 14.90\end{array}$ & $\begin{array}{c}\text { GC: } \\
75.10 \pm 9.60\end{array}$ & & & & \\
\hline & & $\begin{array}{c}\text { GI: } \\
52.20 \pm 15.20\end{array}$ & $\begin{array}{c}\text { Gl: } \\
78.50 \pm 11.50\end{array}$ & & & & \\
\hline & \multirow{6}{*}{$\begin{array}{l}\text { Encuesta de satisfacción } \\
\text { postintervención }\end{array}$} & \multirow{6}{*}{-} & GI: & \multirow{6}{*}{-} & \multirow{6}{*}{-} & \multirow{6}{*}{-} & \multirow{6}{*}{-} \\
\hline & & & $\begin{array}{c}\text { Muy en } \\
\text { desacuerdo: } \\
1.74 \%\end{array}$ & & & & \\
\hline & & & $\begin{array}{l}\text { En desacuer- } \\
\text { do: } 0.24 \%\end{array}$ & & & & \\
\hline & & & $\begin{array}{l}\text { Incierto: } \\
2.75 \%\end{array}$ & & & & \\
\hline & & & $\begin{array}{l}\text { Acuerdo: } \\
31.26 \%\end{array}$ & & & & \\
\hline & & & $\begin{array}{c}\text { Altamente } \\
\text { en acuerdo: } \\
64.01 \%\end{array}$ & & & & \\
\hline & Conocimiento de servicios a pacientes & GC: $4.0 \pm 1.40$ & GC: $5.8 \pm 1.60$ & $\mathrm{C}^{0}$ & & $0 \Omega 6$ & 126 \\
\hline & en comunidad & GI: $3.8 \pm 1.40$ & GI: $5.7 \pm 1.60$ & 0 & - & -0.06 & 1.26 \\
\hline Ellovet al & Confianza on contar una hictoria & GC: $5.7 \pm 1.40$ & GC: $6.2 \pm 1.80$ & : & - & 038 & 002 \\
\hline & Cominama ent comal una mistona & GI: $5.5 \pm 1.50$ & GI: $6.8 \pm 1.30$ & • & - & 0.30 & 0.92 \\
\hline & Facilidad de adoptar un enfoque centra- & GC: $6.0 \pm 1.20$ & GC: $6.8 \pm 1.40$ & 0 & - & 007 & 044 \\
\hline & do en el paciente & GI: $6.3 \pm 1.30$ & GI: $6.9 \pm 1.40$ & & & 0.07 & \\
\hline
\end{tabular}




\begin{tabular}{|c|c|c|c|c|c|c|c|}
\hline \multirow[b]{2}{*}{ Autor(es) } & \multirow[b]{2}{*}{ Variables } & \multirow{2}{*}{$\begin{array}{l}\text { Preinter- } \\
\text { vención } \\
\quad \pm \mathrm{SD}\end{array}$} & \multirow{2}{*}{$\begin{array}{l}\text { Postinter- } \\
\text { vención } \\
\pm S D\end{array}$} & \multicolumn{2}{|c|}{$\begin{array}{c}\text { Diferencias } \\
\text { significativas }\end{array}$} & \multicolumn{2}{|c|}{ Cohen's d (post) } \\
\hline & & & & $\begin{array}{l}\text { Intra } \\
\text { grupo }\end{array}$ & $\begin{array}{l}\text { Inter } \\
\text { grupo }\end{array}$ & $\begin{array}{l}\text { Inter } \\
\text { grupo }\end{array}$ & $\begin{array}{l}\text { Intra } \\
\text { grupo } \\
(\mathrm{Gl})\end{array}$ \\
\hline & \multirow{2}{*}{ Habilidades de comunicación } & GC: $5.7 \pm 1.30$ & GC: $6.2 \pm 1.70$ & \multirow{2}{*}{$\bullet$} & \multirow{2}{*}{ - } & \multirow{2}{*}{0.40} & \multirow{2}{*}{0.80} \\
\hline & & Gl: $5.8 \pm 1.30$ & GI: $6.8 \pm 1.20$ & & & & \\
\hline & \multirow{2}{*}{ Habilidad para detectar depresión } & GC: $6.2 \pm 1.40$ & GC: $6.2 \pm 1.90$ & \multirow{2}{*}{$\bullet$} & \multirow{2}{*}{-} & \multirow{2}{*}{0.63} & \multirow{2}{*}{0.92} \\
\hline & & GI: $6.0 \pm 1.40$ & Gl: $7.2 \pm 1.20$ & & & & \\
\hline & Calidad de atención de general practi- & GC: $6.1 \pm 1.30$ & GC: $7.1 \pm 1.30$ & \multirow{2}{*}{$\circ$} & \multirow{2}{*}{-} & \multirow{2}{*}{-0.72} & \multirow{2}{*}{0.17} \\
\hline & laria especializada & GI: $5.3 \pm 2.40$ & GI: $5.7 \pm 2.40$ & & & & \\
\hline & \multirow{2}{*}{ Interés en la práctica general } & GC: $5.2 \pm 2.20$ & GC: $5.8 \pm 2.50$ & \multirow{2}{*}{$\circ$} & \multirow{2}{*}{-} & \multirow{2}{*}{-0.03} & \multirow{2}{*}{0.16} \\
\hline & & Gl: $5.3 \pm 2.40$ & Gl: $5.7 \pm 2.50$ & & & & \\
\hline \multirow{3}{*}{$\begin{array}{l}\text { Jansson } \\
\text { et al. }\end{array}$} & \multirow{2}{*}{$\begin{array}{l}\text { Conocimiento en el uso de instrumentos } \\
\text { de ventilación }\end{array}$} & $\begin{array}{c}\text { GC: } \\
18.60 \pm 3.25\end{array}$ & $\begin{array}{c}\text { GC: } \\
19.00 \pm 2.99\end{array}$ & \multirow[t]{2}{*}{$\bullet$} & \multirow[t]{2}{*}{-} & \multirow[t]{2}{*}{1.25} & 0.47 \\
\hline & & GI: $21.67 \pm 2.38$ & $\begin{array}{c}\text { Gl: } \\
23.08 \pm 3.50\end{array}$ & & & & \\
\hline & $\begin{array}{l}\text { Habilidades en el uso de instrumentos } \\
\text { de ventilación }\end{array}$ & $\begin{array}{c}\text { GC: } \\
29.33 \pm 3.92 \\
\text { GI: } 28.07 \pm 3.85\end{array}$ & $\begin{array}{c}\text { GC: } \\
29.50 \pm 2.84 \\
\text { Gl: } \\
31.85 \pm 3.31 \\
\end{array}$ & $\bullet$ & - & 0.76 & 1.05 \\
\hline & Actitudes & & GC: 7.20 & - & - & - & - \\
\hline & fecticules & & GI: 8.20 & 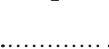 & 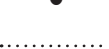 & 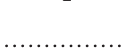 & \\
\hline & Insnección & & GC: 6.70 & & 0 & & \\
\hline & mspeccion & & GI: 7.10 & - & 0 & - & - \\
\hline Janjua A & Examinación bimanual & & GC: 5.80 & - & : & - & - \\
\hline & & & GI: 6.70 & - & 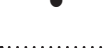 & - & - \\
\hline & Fondo de saco de Douglas & & GC: 5.70 & & ? & - & - \\
\hline & 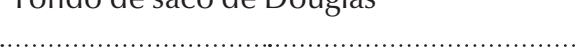 & & GI: 6.30 & 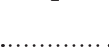 & 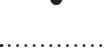 & 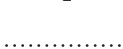 & 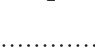 \\
\hline & Fxaminación del esnéculo & & GC: 5.80 & & 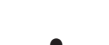 & & \\
\hline & Examinacion der especuro & & GI: 6.80 & - & 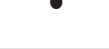 & - & - \\
\hline & & & & $\begin{array}{l}\text { LTI vs } \\
\text { CG: } \circ\end{array}$ & & & \\
\hline & Habilidades técnicas & - & - & $\begin{array}{l}\text { EAI vs } \\
\text { CG: } \circ\end{array}$ & & & \\
\hline & & & & LTI vs & & & \\
\hline Kessler CS. & & & & EAI: $\circ$ & - & - & - \\
\hline et al. & & & & LTI vs & 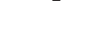 & 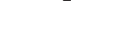 & 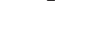 \\
\hline & & & CG: 9.17 & CG: $\bullet$ & & & \\
\hline & Desempeño de la consulta & - & LTI: 10.50 & $\begin{array}{l}\text { EAI vs } \\
\text { CG: } \bullet\end{array}$ & & & \\
\hline & & & EAI: 10.26 & $\begin{array}{l}\text { LTI vs } \\
\text { EAI: } 0\end{array}$ & & & \\
\hline
\end{tabular}




\begin{tabular}{|c|c|c|c|c|c|c|c|c|}
\hline \multirow[b]{2}{*}{ Autor(es) } & \multirow{2}{*}{\multicolumn{2}{|c|}{ Variables }} & \multirow{2}{*}{$\begin{array}{l}\text { Preinter- } \\
\text { vención } \\
\quad \pm \text { SD }\end{array}$} & \multirow{2}{*}{$\begin{array}{l}\text { Postinter- } \\
\text { vención } \\
\pm S D\end{array}$} & \multicolumn{2}{|c|}{$\begin{array}{l}\text { Diferencias } \\
\text { significativas }\end{array}$} & \multicolumn{2}{|c|}{ Cohen's d (post) } \\
\hline & & & & & $\begin{array}{l}\text { Intra } \\
\text { grupo }\end{array}$ & $\begin{array}{l}\text { Inter } \\
\text { grupo }\end{array}$ & $\begin{array}{l}\text { Inter } \\
\text { grupo }\end{array}$ & $\begin{array}{c}\text { Intra } \\
\text { grupo } \\
\text { (GI) }\end{array}$ \\
\hline \multirow{6}{*}{ Kesten } & \multicolumn{2}{|c|}{ Habilidades de comunicación } & $\begin{array}{l}\text { GC: } 60.4 \pm 15.9 \\
\text { GI: } 63.9 \pm 12.8\end{array}$ & $\begin{array}{l}\text { GC: } 84.7 \pm 11.5 \\
\text { GI: } 85.7 \pm 9.4\end{array}$ & ○ & - & 0.09 & 1.94 \\
\hline & \multirow{5}{*}{$\begin{array}{l}\text { Ejecución de la } \\
\text { comunicación }\end{array}$} & Componente 1 & - & $\begin{array}{l}\text { GC: } 53 \\
\text { GI: } 54\end{array}$ & ० & - & - & - \\
\hline & & Componente 2 & - & $\begin{array}{l}\text { GC: } 42 \\
\text { GI: } 47\end{array}$ & $\circ$ & - & - & - \\
\hline & & Componente 3 & - & $\begin{array}{l}\text { GC: } 23 \\
\text { GI: } 35\end{array}$ & $\bullet$ & - & - & - \\
\hline & & Componente 4 & - & $\begin{array}{l}\text { GC: } 37 \\
\text { GI: } 42\end{array}$ & ० & - & - & - \\
\hline & & Componente 5 & - & $\begin{array}{l}\text { GC: } 39 \\
\text { GI: } 42\end{array}$ & $\circ$ & - & - & - \\
\hline $\begin{array}{l}\text { Knowles } \\
\text { et al. }\end{array}$ & \multicolumn{2}{|c|}{$\begin{array}{l}\text { Habilidades y conocimiento de la } \\
\text { comunicación }\end{array}$} & - & $\begin{array}{l}\text { GC: } 60.4 \\
\text { GI: } 73.1\end{array}$ & $\bullet$ & - & - & - \\
\hline \multirow{2}{*}{$\begin{array}{l}\text { Koponen } \\
\text { et al. }\end{array}$} & \multicolumn{2}{|c|}{$\begin{array}{l}\text { Actitudes positivas al aprendizaje de las } \\
\text { habilidades de comunicación }\end{array}$} & $\begin{array}{l}\text { EOA: } 45 \\
\text { PS: } 45 \\
\text { RP: } 43\end{array}$ & $\begin{array}{c}\text { EOA: } 49 \\
\text { PS: } 46 \\
\text { RP: } 45\end{array}$ & ○ & $\begin{array}{l}\text { EOA: • } \\
\text { PS: • } \\
\text { RP: } \bullet\end{array}$ & - & - \\
\hline & \multicolumn{2}{|c|}{$\begin{array}{l}\text { Actitudes negativas al aprendizaje de las } \\
\text { habilidades de comunicación }\end{array}$} & $\begin{array}{c}\text { EOA: } 29.5 \\
\text { PS: } 29.5 \\
\text { RP: } 32\end{array}$ & $\begin{array}{l}\text { EOA: } 26 \\
\text { PS: } 28 \\
\text { RP: } 30\end{array}$ & ० & $\begin{array}{l}\text { EOA: • } \\
\text { PS: } \\
\text { RP: ○ }\end{array}$ & - & - \\
\hline \multirow{6}{*}{ Kin \& Shin } & \multicolumn{2}{|c|}{ Conocimiento (Emergencias neonatal) } & $\begin{array}{l}\text { GC: } 6.00 \pm 1.07 \\
\text { GI: } 5.42 \pm 0.95\end{array}$ & $\begin{array}{l}\text { GC: } 8.17 \pm 0.62 \\
\text { GI: } 8.86 \pm 0.55\end{array}$ & $\bullet$ & $\begin{array}{l}\text { CG: • } \\
\text { IG: • }\end{array}$ & 1.17 & 4.43 \\
\hline & \multicolumn{2}{|c|}{ Actitud (Emergencias neonatal) } & $\begin{array}{l}\text { GC: } 5.96 \pm 2.69 \\
\text { GI: } 6.60 \pm 1.16\end{array}$ & $\begin{array}{l}\text { GC: } 7.71 \pm 1.83 \\
\text { GI: } 8.48 \pm 1.12\end{array}$ & $\bullet$ & $\begin{array}{l}\text { CG: } \\
\text { IG: }\end{array}$ & 0.50 & 1.64 \\
\hline & \multicolumn{2}{|c|}{ Habilidades (Emergencias neonatal) } & $\begin{array}{l}\text { GC: } 6.44 \pm 1.45 \\
\text { GI: } 6.89 \pm 1.29\end{array}$ & $\begin{array}{l}\text { GC: } 6.71 \pm 1.53 \\
\text { Gl: } 8.24 \pm 1.45\end{array}$ & $\bullet$ & $\begin{array}{l}\text { CG: } \\
\text { IG: }\end{array}$ & 1.02 & 0.98 \\
\hline & \multicolumn{2}{|c|}{ Conocimiento (Emergencias adulto) } & $\begin{array}{l}\text { GC: } 5.15 \pm 1.14 \\
\text { GI: } 4.36 \pm 0.87\end{array}$ & $\begin{array}{l}\text { GC: } 7.46 \pm 0.79 \\
\text { GI: } 9.02 \pm 0.32\end{array}$ & $\bullet$ & $\begin{array}{l}\text { CG:• } \\
\text { IG:• }\end{array}$ & 2.58 & 7.10 \\
\hline & \multicolumn{2}{|c|}{ Actitud (Emergencias adulto) } & $\begin{array}{l}\text { GC: } 5.54 \pm 1.91 \\
\text { GI: } 5.60 \pm 1.19\end{array}$ & $\begin{array}{l}\text { GC: } 6.71 \pm 1.55 \\
\text { GI: } 8.88 \pm 0.73\end{array}$ & $\bullet$ & $\begin{array}{l}\text { CG:• } \\
\text { IG:• }\end{array}$ & 1.79 & 3.32 \\
\hline & \multicolumn{2}{|c|}{ Habilidades (Emergencias adulto) } & $\begin{array}{l}\text { GC: } 6.14 \pm 1.29 \\
\text { GI: } 6.43 \pm 1.19\end{array}$ & $\begin{array}{l}\text { GC: } 6.64 \pm 1.49 \\
\text { GI: } 8.06 \pm 1.50\end{array}$ & $\bullet$ & $\begin{array}{l}\text { CG: • } \\
\text { IG:• }\end{array}$ & 0.94 & 1.20 \\
\hline
\end{tabular}




\begin{tabular}{|c|c|c|c|c|c|c|c|c|}
\hline \multirow[b]{2}{*}{ Autor(es) } & \multirow{2}{*}{\multicolumn{2}{|c|}{ Variables }} & \multirow{2}{*}{$\begin{array}{l}\text { Preinter- } \\
\text { vención } \\
\quad \pm S D\end{array}$} & \multirow{2}{*}{$\begin{array}{l}\text { Postinter- } \\
\text { vención } \\
\pm S D\end{array}$} & \multicolumn{2}{|c|}{$\begin{array}{l}\text { Diferencias } \\
\text { significativas }\end{array}$} & \multicolumn{2}{|c|}{ Cohen's d (post) } \\
\hline & & & & & $\begin{array}{l}\text { Intra } \\
\text { grupo }\end{array}$ & $\begin{array}{l}\text { Inter } \\
\text { grupo }\end{array}$ & $\begin{array}{l}\text { Inter } \\
\text { grupo }\end{array}$ & $\begin{array}{c}\text { Intra } \\
\text { grupo } \\
\text { (GI) }\end{array}$ \\
\hline \multirow{4}{*}{$\begin{array}{l}\text { Schlegel } \\
\text { et al. }\end{array}$} & \multirow{2}{*}{ Autoeficacia } & Conocimiento & - & $\begin{array}{l}\text { GC: } 37.30 \\
\text { GI: } 39.90\end{array}$ & ? & - & - & - \\
\hline & & Habilidad práctica & - & $\begin{array}{l}\text { GC: } 35.10 \\
\text { GI: } 35.80\end{array}$ & ० & - & - & - \\
\hline & $\begin{array}{l}\text { Perspectiva c } \\
\text { de la comun }\end{array}$ & $\begin{array}{l}\text { aciente sobre la calidad } \\
\text { ión }\end{array}$ & - & - & ○ & - & - & - \\
\hline & $\begin{array}{l}\text { Perspectiva c } \\
\text { dad de la cor }\end{array}$ & $\begin{array}{l}\text { upervisor sobre la cali- } \\
\text { icación }\end{array}$ & - & - & $\bullet$ & - & - & - \\
\hline \multirow{4}{*}{$\begin{array}{l}\text { Zavertnik } \\
\text { et al. }\end{array}$} & Introducción & & - & $\begin{array}{l}\text { GC: } 0.9 \pm 1.0 \\
\text { GI: } 1.1 \pm 1.0\end{array}$ & o & - & 0.20 & - \\
\hline & Recopilaciór & información & - & $\begin{array}{l}\text { GC: } 5.0 \pm 1.2 \\
\text { GI: } 6.1 \pm 2.0\end{array}$ & $\bullet$ & - & 0.66 & - \\
\hline & Impartición & fformación & - & $\begin{array}{l}\text { GC: } 5.3 \pm 1.6 \\
\text { GI: } 5.8 \pm 1.7\end{array}$ & o & - & 0.30 & - \\
\hline & Aclaración d & jetivos y expectativas & - & $\begin{array}{l}\text { GC: } 6.2 \pm 1.4 \\
\text { Gl: } 6.6 \pm 1.8\end{array}$ & ० & - & 0.24 & - \\
\hline
\end{tabular}

ET: enseñanza tradicional; EA: enseñanza autodidacta; EO: enseñanza online; GC: grupo control; GI: grupo intervención; FB: fidelidad baja; FM: fidelidad media; FA: fidelidad baja; EOA: educación en obra teatral; PS: paciente simulado; RL: role-play

\section{Síntesis de los resultados}

Las 35 variables analizadas en los 14 estudios incluidos en esta revisión sistemática ( $\geq 5$ puntos en la escala PEDro) se organizaron en cuatro categorías: adquisición de conocimiento, aprendizaje de la práctica clínica, autoeficacia y desarrollo de habilidades de comunicación. Se observaron diferencias significativas intergrupo en el $40 \%$ de las variables donde se evaluó ( $d$ de Cohen =1.25-0.32) (Aper et al., 2012; Elley et al., 2012; Jansson et al., 2014; Kesten, 2011; Knowles et al., 2001; Lee Chin et al., 2014; Schlegel et al., 2012; Zavertnik et al., 2010) e intragrupo del grupo experimental en el $66.7 \%$ de las variables donde se evaluó $\left(\mathrm{d}\right.$ de Cohen $=0.42^{-}$ o.70) (Aper et al., 2012; Becker et al., 2006; Koponen et al., 2012). Estos resultados confirman la efectividad de la simulación de pacientes estandarizados como un método para enseñar conocimiento teórico relevante para la práctica clínica y mejorar el desempeño en los roles médicos y de enfermería.

\section{VARIABLES DE RESULTADO}

\section{Adquisición de conocimiento}

Esta variable se evaluó antes y después de la intervención en ocho estudios (Becker et al., 2006; Brady et al., 2015; Elley et al., 2012; Jansson et al., 2014; Kesten, 2011; Kim \& Shin, 2016; Knowles et al., 2001; Lee Chin et al., 2014), pero Knowles et al. (Knowles et al., 2001) y Brady et al. (Brady et al., 2015) solo midieron las variables de conocimiento después de la intervención.

Cuatro estudios encontraron diferencias significativas entre los grupos después de la intervención [d de Cohen=1.25 (Jansson et al., 2014); d de Cohen=0.32 (Jansson et al., 2014; Knowles et al., 2001; Lee Chin et al., 2014); d de Cohen $=0.80$ y d de Cohen $=0.79$ (Brady et al., 2015); d de Cohen=1.17 y d de Cohen=2.58 (Kim \& Shin, 2016) (Tabla 3); sin embargo, los otros estudios (Becker 
et al., 2006; Elley et al., 2012; Kesten, 2011), no mostraron diferencias significativas, a pesar de utilizar participantes similares (estudiantes de pregrado o posgrado), el mismo método de enseñanza (simulación de pacientes humanos) y protocolos similares (sesiones de simulación que duran 10-30 minutos). La ausencia de diferencias significativas en estos estudios (Becker et al., 2006; Elley et al., 2012; Kesten, 2011) puede deberse al hecho de que estos tres estudios evaluaron la adquisición de conocimiento sobre técnicas de comunicación, mientras que los dos estudios que encontraron diferencias significativas posteriores a la intervención evaluaron la adquisición de conocimiento sobre la práctica con el paciente (Jansson et al., 2014; Knowles et al., 2001; Lee Chin et al., 2014).

Después de una comparación intragrupo de esta variable posterior a la intervención, dos estudios informaron de un cambio en el grupo de aprendizaje mediante simulación $(\mathrm{d}=0.70)$ (Becker et al., 2006); $(\mathrm{d}=4.43$, $\mathrm{d}=7.10$ )(Kim \& Shin, 2016) (Tabla 3). Este grupo vio un video explicativo antes de hacer el ejercicio de simulación con pacientes estandarizados, por lo que su mejora en el aprendizaje puede deberse al efecto combinado de ambas técnicas (visualización explicativa de video y simulación de pacientes humanos), resultados similares a los encontrados en el estudio de Kim \& Shin (2016) en el que se combinaron diversas técnicas educativas.

Los resultados de las comparaciones intergrupo e intragrupo en los estudios analizados confirman que la simulación con pacientes estandarizados es un método más efectivo para aprender o mejorar el conocimiento sobre la práctica de la medicina y la enfermería que los enfoques tradicionales. La efectividad de la simulación con pacientes estandarizados parece mejorar cuando los estudiantes ven un video explicativo de antemano.

\section{Aprendizaje de la práctica clínica}

Se encontraron diferencias significativas entre los grupos en los siete estudios que lo evaluaron (Aper et al., 2012; Brady et al., 2015; Elley et al., 2012; Janjua et al., 2017; Jansson et al., 2014; Kessler et al., 2015), los tamaños del efecto fueron desde $\mathrm{d}=0.38$ (Elley et al., 2012), demostrando que la simulación de pacientes humanos es más efectiva que la enseñanza tradicional como una forma de entrenar a los estudiantes en el aprendizaje de la medicina y la práctica de enfermería. Es importante destacar, sin embargo, que Elley et al. (2012) no encontraron diferencias entre grupos significativas en diversas variables de aprendizaje de la práctica clínica, como fueron "Facilidad con la que se adopta un enfoque centrado en el paciente", "Calidad de la atención del médico generalista en comparación con la atención hospitalaria especializada" e "Interés en la práctica general", similar a Kessler et al. (2015), en el que no se encontraron diferencias significativas en "habilidades técnicas". A diferencia de Aper et al. (2012) y Jansson et al. (2014), Elley et al. (2012) no realizó la sesión de información después de la simulación. Ya que esta etapa se ha identificado como una parte fundamental del proceso de aprendizaje mediante la simulación (Levett-Jones \& Lapkin, 2014), la falta de esta puede ser la responsable de la ausencia de cambios en algunas de las variables de aprendizaje de la práctica clínica en el estudio de Elley et al. (2012).

Por otro lado, Aper et al. (2012) analizaron los cambios intragrupo en el aprendizaje de las variables de la práctica clínica y no encontraron mejoría en el grupo experimental después de la simulación con paciente humano.

Por otro lado, Aper et al. (2012) analizaron los cambios intragrupo en las variables de aprendizaje de la práctica clínica y no encontraron mejoría en el grupo experimental después de la simulación con pacientes estandarizados.

\section{Autoeficacia}

De los cinco estudios que evaluaron la autoeficacia (Aper et al., 2012; Becker et al., 2006; Janjua et al., 2017; Lee Chin et al., 2014; Schlegel et al., 2012), sólo un estudio (Aper et al., 2012) midió las variables relacionadas antes y después de la intervención. Después de analizar las diferencias significativas intragrupo en el estudio de Aper et al. (2012), se encontró que el grupo de simulación mostró mejoras en las variables de autoeficacia después del entrenamiento $(\mathrm{d}=0.42 \mathrm{yd}=0.58)$, mientras que los grupos de entrenamiento tradicional y en línea no lo hicieron. Estos resultados sugieren que la simulación con pacientes estandarizados es más efectiva que el entrenamiento tradicional como un método para mejorar la autoeficacia relacionada con el aprendizaje de los estudiantes de medicina y se logró a pesar de que el entrenamiento tradicional se llevó a cabo durante un período de tiempo más largo y más sesiones 
de entrenamiento. Se necesita más investigación para confirmar este hallazgo.

\section{Desarrollo de habilidades de comunicación}

Esta categoría de resultados agrupa 15 variables utilizadas como indicadores de varios aspectos de las habilidades de comunicación en siete de los estudios incluidos en la revisión sistemática (Becker et al., 2006; Elley et al., 2012; Kessler et al., 2015; Kesten, 2011; Koponen et al., 2012; Schlegel et al., 2012; Zavertnik et al., 2010) (Tabla 2). Hubo diferencias significativas entre grupos en cinco de las variables (Elley et al., 2012; Kessler et al., 2015; Kesten, 2011; Schlegel et al., 2012; Zavertnik et al., 2010), con valores de la d de Cohen oscilando entre 0.40 (Elley et al., 2012) y 0.66 (Zavertnik et al., 2010) (Tabla 3). En el caso de dos de las 15 variables de habilidades de comunicación, hubo efectos intragrupo en los grupos que realizaron algún tipo de simulación (simulación con pacientes estandarizados y teatro en educación) (Koponen et al., 2012). La descomposición de las habilidades de comunicación puede ser la razón por la cual, después de analizar la comparación inter e intragrupo, no se encontraron resultados concluyentes. Elley et al. (2012) evaluó la habilidad de comunicación como una variable única y observó diferencias significativas en el grupo experimental después de llevar a cabo la simulación con paciente estandarizado (Tabla 3).

\section{Fortalezas y limitaciones}

La validez interna de los estudios incluidos en esta revisión sistemática varió de 5 a 7 puntos en la escala PEDro (Tabla 1). Este criterio se usó para evitar la inclusión de estudios con baja validez interna, reduciendo la solidez de los resultados generales. También vale la pena mencionar que la homogeneidad de las variables nos permitió agruparlas en cuatro categorías, relacionadas con aspectos del aprendizaje a través de la simulación. Se debe enfatizar, a su vez, que todos los estudios incluidos compararon el método de simulación con otro método (grupo de control). Entre las principales debilidades de esta revisión están la falta de estudios que utilicen la simulación con pacientes estandarizados en más disciplinas sanitarias, especialmente en terapia ocupacional y la falta de consistencia en la aplicación del procedimiento esta metodología. Estas debilidades significan que los resultados de esta revisión sistemática no pueden generalizarse o extrapolarse a otras disciplinas de ciencias de la salud.

\section{Aplicabilidad práctica}

Los resultados de la presente revisión sistemática sugieren el uso de los vídeos explicativos al comienzo de la sesión docente, los cuales podrían ser incorporados en la docencia de cualquier disciplina sanitaria. La inclusión de estos vídeos mejora el aprendizaje del alumno visualizando en el vídeo el uso práctico del material que el docente va a impartir. Además de ello, se debería añadir también la realización de un informe final por parte del alumno sobre las competencias adquiridas. Por otro lado, a pesar de que la simulación con pacientes estandarizados no ha mostrado una mayor efectividad que los métodos tradicionales, este método podría ser añadido en la docencia ya que modifica el método didáctico habitual y permite el aprendizaje en un contexto y situación similar al de la práctica clínica.

\section{Futuras líneas de investigación}

En la actualidad no existen estudios que hayan diseñado, desarrollado y aplicado la simulación con pacientes estandarizados en las titulaciones de terapia ocupacional, a pesar de ser una metodología ampliamente empleada en esta formación. Debido al papel activo del usuario en el desempeño del rol del terapeuta ocupacional en el ámbito clínico, la creación de este espacio simulado con una persona podría permitir a los estudiantes del grado de terapia ocupacional la adquisición de algunas habilidades clínicas de una forma más natural. Para conocer los beneficios que la simulación podría aportar, frente a las metodologías tradicionales, en los estudiantes del grado de terapia ocupacional, sería necesario la realización de estudios que aportasen evidencias en este aspecto.

\section{CONCLUSIÓN}

Los datos alcanzados en la presente revisión sistemática no permiten confirmar que la simulación con pacientes estandarizados aporte un mayor aprendizaje de las habilidades clínicas frente a la metodología tradicional de enseñanza clínica, aunque sí aportan un contexto más próximo a la realidad en el que ponerlas en 
práctica. A su vez, cabe destacar que la simulación es más efectiva cuando en las fases del proceso de aprendizaje se incorporan la visualización de videos explicativos al comienzo de este y la creación de informes en la fase final de resumen y conclusiones. Estudios futuros son necesarios para conocer los beneficios que podría aportar en la titulación de terapia ocupacional.

\section{RefERENCIAS BibLIOGRÁfICAS}

Andrighetti, T. P., Knestrick, J. M., Marowitz, A., Martin, C., \& Engstrom, J. L. (2012). Shoulder dystocia and postpartum hemorrhage simulations: Student confidence in managing these complications. Journal of Midwifery \& Women's Health, 57(1), 55-60. https://doi. org/10.1111/j.1542-2011.2011.00085.x

Aper, L., Reniers, J., Koole, S., Valcke, M., \& Derese, A. (2012). Impact of three alternative consultation training formats on self-efficacy and consultation skills of medical students. Medical Teacher, 34(7), e500-507. https://doi.org/10.3109/0142159X.2012.668627

Becker, K. L., Rose, L. E., Berg, J. B., Park, H., \& Shatzer, J. H. (2006). The teaching effectiveness of standardized patients. The Journal of Nursing Education, 45(4), 103-111. https://doi.org/10.3928/0148483420060401-03

Bingham, A. L., Sen, S., Finn, L. A., \& Cawley, M. J. (2015). Retention of advanced cardiac life support knowledge and skills following high-fidelity mannequin simulation training. American Journal of Pharmaceutical Education, 79(1), 12. https://doi.org/10.5688/ ajpe79112

Brady, S., Bogossian, F., \& Gibbons, K. (2015). The effectiveness of varied levels of simulation fidelity on integrated performance of technical skills in midwifery students-A randomised intervention trial. Nurse Education Today, 35(3), 524-529. https://doi. org/10.1016/j.nedt.2014.11.005

Elizondo-Omaña, R. E., Morales-Gómez, J. A., Guzmán, S. L., Hernández, I. L., Ibarra, R. P., \& Vilchez, F. C. (2004). Traditional teaching supported by computer-assisted learning for macroscopic anatomy. Anatomical Record. Part B, New Anatomist, 278(1), 18-22. https:// doi.org/10.1002/ar.b.20019

Elley, C. R., Clinick, T., Wong, C., Arroll, B., Kennelly, J., Doerr, H., Moir, F., Fishman, T., Moyes, S. A., \& Kerse, N. (2012). Effectiveness of simulated clinical teaching in general practice: Randomised controlled trial. Journal of Primary Health Care, 4(4), 281-287.

Gordon, C. J., Frotjold, A., Fethney, J., Green, J., Hardy, J., Maw, M., \& Buckley, T. (2013). The effectiveness of simulation-based blood pressure training in preregistration nursing students. Simulation in Healthcare: Journal of the Society for Simulation in Healthcare, 8(5), 335-340. https://doi.org/10.1097/SIH.0b013e3182a15fa7

Guarino, S., Leopardi, E., Sorrenti, S., De Antoni, E., Catania, A., \& Alagaratnam, S. (2014). Internet-based versus traditional teaching and learning methods. The Clinical Teacher, 17(6), 449-453. https:// doi.org/10.1111/tct.12191

Hong, C. K., Park, S. O., Jeong, H. H., Kim, J. H., Lee, N. K., Lee, K. Y., Lee, Y., Lee, J. H., \& Hwang, S. Y. (2014). The most effective rescuer's position for cardiopulmonary resuscitation provided to patients on beds: A randomized, controlled, crossover mannequin study. The Journal of Emergency Medicine, 46(5), 643-649. https://doi. org/10.1016/j.jemermed.2013.08.085

Janjua, A., Smith, P., Chu, J., Raut, N., Malick, S., Gallos, I., Singh, R., Irani, S., Gupta, J. K., Parle, J., \& Clark, T. J. (2017). The effectiveness of gynaecology teaching associates in teaching pelvic examination to medical students: A randomised controlled trial. European Journal of Obstetrics, Gynecology, and Reproductive Biology, 210, 58-63. https://doi.org/10.1016/j.ejogrb.2016.10.006

Jansson, M. M., Ala-Kokko, T. I., Ohtonen, P. P., Meriläinen, M. H., Syrjälä, H. P., \& Kyngäs, H. A. (2014). Human patient simulation education in the nursing management of patients requiring mechanical ventilation: A randomized, controlled trial. American Journal of Infection Control, 42(3), 271-276. https://doi.org/10.1016/j. ajic.2013.11.023

Kessler, C. S., Tadisina, K. K., Saks, M., Franzen, D., Woods, R., Banh, K. V., Bounds, R., Smith, M., Deiorio, N., \& Schwartz, A. (2015). The 5Cs of Consultation: Training Medical Students to Communicate Effectively in the Emergency Department. The Journal of Emergency Medicine, 49(5), 713-721. https://doi.org/10.1016/j. jemermed.2015.05.012

Kesten, K. S. (2011). Role-play using SBAR technique to improve observed communication skills in senior nursing students. The Journal of Nursing Education, 50(2), 79-87. https://doi. org/10.3928/01484834-20101230-02

Kim, S., \& Shin, G. (2016). Effects of nursing process-based simulation for maternal child emergency nursing care on knowledge, attitude, and skills in clinical nurses. Nurse Education Today, 37, 59-65. https://doi.org/10.1016/j.nedt.2015.11.016

Knowles, C., Kinchington, F., Erwin, J., \& Peters, B. (2001). A randomised controlled trial of the effectiveness of combining video role play with traditional methods of delivering undergraduate medical education. Sexually Transmitted Infections, 77(5), 376-380. https:// doi.org/10.1136/sti.77.5.376

Koponen, J., Pyörälä, E., \& Isotalus, P. (2012). Comparing three experiential learning methods and their effect on medical students' attitudes to learning communication skills. Medical Teacher, 34(3), e198-207. https://doi.org/10.3109/0142159X.2012.642828

Lee Chin, K., Ling Yap, Y., Leng Lee, W., \& Chang Soh, Y. (2014). Comparing effectiveness of high-fidelity human patient simulation vs case-based learning in pharmacy education. American Journal of Pharmaceutical Education, 78(8), 153. https://doi.org/10.5688/ ajpe788153

Levett-Jones, T., \& Lapkin, S. (2014). A systematic review of the effectiveness of simulation debriefing in health professional education. 
Nurse Education Today, 34(6), e58-63. https://doi.org/10.1016/j. nedt.2013.09.020

Maher, C. G., Sherrington, C., Herbert, R. D., Moseley, A. M., \& Elkins, M. (2003). Reliability of the PEDro scale for rating quality of randomized controlled trials. Physical Therapy, 83(8), 713-721.

McParland, M., Noble, L. M., \& Livingston, G. (2004). The effectiveness of problem-based learning compared to traditional teaching in undergraduate psychiatry. Medical Education, 38(8), 859-867. https://doi.org/10.1111/j.1365-2929.2004.01818.x

Moher, D., Liberati, A., Tetzlaff, J., Altman, D. G., \& PRISMA Group. (2009). Preferred reporting items for systematic reviews and metaanalyses: The PRISMA statement. PLoS Medicine, 6(7), e1000097. https://doi.org/10.1371/journal.pmed.1000097

Moseley, A. M., Herbert, R. D., Sherrington, C., \& Maher, C. G. (2002). Evidence for physiotherapy practice: A survey of the Physiotherapy Evidence Database (PEDro). The Australian Journal of Physiotherapy, 48(1), 43-49. https://doi.org/10.1016/s0004-9514(14)60281-6

Schlegel, C., Woermann, U., Shaha, M., Rethans, J.-J., \& van der Vleuten, C. (2012). Effects of communication training on real practice performance: A role-play module versus a standardized patient module. The Journal of Nursing Education, 57(1), 16-22. https:// doi.org/10.3928/01484834-20111116-02

Stefaniak, J. E., \& Turkelson, C. L. (2014). Does the sequence of instruction matter during simulation? Simulation in Healthcare: Journal of the Society for Simulation in Healthcare, 9(1), 15-20. https://doi. org/10.1097/SIH.0b013e3182a8336f

Zavertnik, J. E., Huff, T. A., \& Munro, C. L. (2010). Innovative approach to teaching communication skills to nursing students. The Journal of Nursing Education, 49(2), 65-71. https://doi. org/10.3928/01484834-20090918-06 